\title{
Gestión de la continuidad de negocio: caso Ravmar Freight del sector logístico
}

Business continuity management: Ravmar Freight case of the logistics sector

\author{
Eleyn Cano Sotomayor \\ Pontificia Universidad Católica del Perú \\ eleyn.cano@pucp.edu.pe \\ Italo Henry Plaza Aranda \\ Pontificia Universidad Católica del Perú \\ italo.plaza@pucp.edu.pe \\ Evelyn Karina Ramírez Chávez \\ Pontificia Universidad Católica del Perú \\ evelyn.ramirez@pucp.edu.pe
}


La pandemia de COVID-19 demostró la necesidad de la implementación de un sistema de continuidad del negocio en las organizaciones para lidiar con situaciones críticas y eventos que pudiesen frenar sus operaciones. El presente estudio tiene por objetivo proponer el diseño del sistema de gestión de continuidad del negocio (SGCN) de la agencia de carga Ravmar, con base en la norma ISO 22301:2019. El método de trabajo es analítico-descriptivo y de gestión aplicada. Para ello, se realizaron entrevistas virtuales con la Alta Dirección de Ravmar para conocer al detalle sus procesos $y$, después, se analizó toda la información obtenida con la finalidad de determinar el nivel de cumplimiento de la empresa conforme a los requisitos de la norma ISO. Luego del diagnóstico, se desarrolló la propuesta de diseño del SGCN para la organización en mención, bajo la óptica de la norma ISO 22301:2019, asegurando un nivel de cumplimiento del $100 \%$ de los requerimientos señalados por las cláusulas de la norma. Finalmente, se realizó el análisis de viabilidad de la implementación del diseño, concluyendo que la propuesta es viable y que el nivel de cumplimiento que presenta el SGCN de Ravmar, conforme a los requisitos estipulados en la ISO 22301:2019, es de $10 \%$. Este porcentaje demuestra que la organización no cuenta con información relacionada a la continuidad del negocio para los procesos que forman parte del servicio de exportación.

Palabras clave: sistema de gestión de continuidad del negocio, SGCN, ISO 22301:2019, plan de continuidad del negocio, plan de contingencia, gestión de riesgos

The COVID-19 pandemic demonstrated the need for the implementation of a business continuity system in organizations to deal with critical situations and events that could stop their operations. The objective of this study is to design the business continuity management system (BCMS) of the Ravmar freight agency, based on the ISO 22301 :2019 standard. The work method is analytical-descriptive and applied management. To do this, virtual interviews were held with Ravmar's Senior Management to learn about its processes in detail and, then, all the information obtained was analyzed in order to determine the company's level of compliance with the requirements of the ISO standard. After the diagnosis, the proposal for the design of the BCMS was developed for the organization in question, ensuring a level of $100 \%$ compliance of the requirements indicated by the clauses of the rule. Finally, the feasibility analysis of the design implementation was carried out, concluding that the proposal is viable and that the level of compliance presented by the Ravmar BCMS, in accordance with the requirements stipulated in ISO 22301:2019, is of $10 \%$. This percentage shows that the organization does not have information related to business continuity for the processes that are part of the export service.

Keywords: business continuity management system, BCMS, ISO 22301:2019, business continuity plan, contingency plan, risk management 


\section{Introducción}

A lo largo de los años, las empresas se ven afectadas por eventos disruptivos, los cuales interfieren en el desarrollo regular de sus respectivas operaciones. Estos pueden ser eventos como la suspensión del suministro del servicio de luz o, incluso, una pandemia como la de COVID-19.

A inicios de 2020 se experimentó a nivel mundial el inicio de la pandemia, un evento que ninguna organización tenía previsto. Desde enero de 2020 hasta la quincena de junio de 2021, el Perú registró dos millones de personas infectadas y casi doscientas mil personas fallecidos (WHO, 2021). Asimismo, la pandemia paralizó tanto la economía mundial como la nacional. Según el Foro Económico Mundial (2020), el tercer país más afectado de Latinoamérica fue el Perú debido a que una gran parte de su población subsiste con ingresos diarios, lo cual se aúna al deficiente sistema nacional de salud y los inexistentes programas de beneficios como el seguro de desempleo, entre otras variables.

En Perú hubo varias industrias paralizadas debido a la pandemia, siendo una de ellas la del comercio exterior. Desafortunadamente, desde antes de que tenga lugar la pandemia en el país, el sector del comercio exterior presentaba aspectos por mejorar en procesos como compras, abastecimiento y transporte, entre otros, tal como lo señala el indicador de desempeño logístico (LPI) del Banco Mundial. Dicho indicador clasificó al Perú en el puesto 84 de 160 países (World Bank Group, 2018), además de ocupar el cuarto y último puesto respecto a los países de la Alianza del Pacífico, posición que se explica en razón de los insuficientes esfuerzos del Estado por contribuir a la mejora de los seis componentes del LPI (Cordova \& Taquía, 2017). Inclusive, si se compara entre los países de la Alianza del Pacífico, Perú es el único que en 2019 crece en menor porcentaje respecto al año anterior, lo cual provocó que el país descienda en el ranking de competitividad global (Cordova \& Wong, 2020).

La agencia de carga Ravmar, sujeto de estudio del presente artículo, es un operador logístico con más de diez años de experiencia en el sector del comercio exterior que brinda servicios de importación, exportación y postventa. Esta empresa no ha sido ajena a la afectación por la pandemia de COVID-19, ya que se vio fuertemente impactada a nivel económico. La pandemia generó el cese de operaciones de su aliado principal: las aerolíneas, con lo cual sus operaciones descendieron al 16,67 \%. Así, la compañía pasó de percibir, en promedio, USD 3000 mensuales, que equivalen a cinco operaciones diarias, a USD 500 mensuales, que equivalen a una operación diaria. Esto constituyó un efecto negativo muy fuerte para Ravmar, lo que la colocó en una posición de insostenibilidad en el tiempo, exponiéndola a tener el mismo destino que otras empresas durante la pandemia: el cese de operaciones.

Para no enfrentar el cese de las operaciones de manera parcial y/o total, afectando así a las partes interesadas pertinentes (proveedores, trabajadores y clientes, entre otros), es crucial contar con un plan de acción frente a cada tipo de evento disruptivo, el cual se conoce como plan de continuidad del negocio (Torabi et al., 2016). De esta manera, se propuso el diseño de un sistema de gestión de continuidad del negocio (SGCN) en la agencia de carga Ravmar, acorde con los requisitos y lineamientos de la norma 
ISO 22301:2019, respecto al servicio de exportación. Cabe precisar que esta empresa fue elegida por las consideraciones económicas de nuestro sujeto de estudio.

La metodología utilizada fue descriptiva puesto que tiene como finalidad especificar las características del proceso, que en el caso del presente artículo sería el del servicio de exportación que ofrece la agencia de carga con base en la ISO 22301:2019 (Pasco \& Ponce, 2018, p. 43). La mencionada propuesta está estructurada en cuatro partes. Primero, se desarrolla el marco teórico, el cual está compuesto por tres ejes temáticos relacionados al presente documento: gestión de la continuidad del negocio, gestión por procesos y gestión de riesgos. Segundo, se presenta el marco metodológico utilizado para llevar a cabo la propuesta de diseño del sistema integrado de gestión. Tercero, se presenta la propuesta de diseño de un SGCN, así como la viabilidad de su implementación en la agencia de carga Ravmar. Y, por último, se finaliza con las conclusiones del estudio.

La propuesta del presente artículo genera aportes tanto a nivel social y organizacional como académico. Respecto al aporte social, se logran satisfacer las necesidades de los stakeholders; es decir, de los clientes que necesitan de los servicios de Ravmar, de los proveedores que necesitan del flujo de pagos y de los trabajadores que conservan su trabajo, entre otros. Asimismo, genera un aporte organizacional al brindar información suficiente a Ravmar sobre cómo actuar ante eventos disruptivos e, inclusive, conseguir una certificación internacional con una casa acreditadora. Por último, representa un aporte al sector del comercio exterior en el que se encuentran las agencias de carga al proponer un plan de gestión de continuidad del negocio que brinda un modelo replicable en organizaciones similares que, además, sirve como una nueva fuente de conocimiento, ya que no existen estudios como el presente artículo.

\section{Marco teórico}

\subsection{Gestión de la continuidad del negocio}

La gestión de la continuidad del negocio es un proceso que identifica las potenciales amenazas a una organización y el impacto que estas podrían causar en las operaciones del negocio si llegan a materializarse. De igual forma, proporciona la base para desarrollar la resiliencia organizacional y brindar una respuesta efectiva ante las potenciales amenazas (Castro, 2013). Esta resiliencia organizacional implica la capacidad de adaptabilidad de las organizaciones ante cambios, amenazas y eventos disruptivos (Pacheco-Mangas et al., 2020). En ese sentido, como menciona Frikha (2021), la gestión de la continuidad del negocio brinda planes de continuidad del negocio para frenar las interrupciones y disrupciones. Adicionalmente, cabe precisar que la continuidad del negocio permite a la organización seguir entregando los productos y servicios dentro de un tiempo razonable ante algún evento disruptivo (ISO, 2019).

De acuerdo con Quevedo (2012), existen una gran cantidad de modelos de continuidad del negocio, estando entre los más resaltantes el modelo británico BS-25999 Sistema de Gestión de Continuidad del Negocio, que brinda un guía de recomendaciones, pasos a seguir, requerimientos y buenas prácticas para el correcto funcionamiento de un SGCN. Este modelo constituye la base para otros que han sido desarrollados 
posteriormente. También existe el modelo para el planeamiento de gestión de crisis e incidentes, el cual plantea seis componentes: el marco de referencia, el equipo de la gestión de crisis e incidentes, el centro de operaciones para emergencias, los procedimientos de comunicación en crisis, el software de gestión de continuidad del negocio y el ejercicio de los procedimientos de gestión de la crisis. Luego de conocer los diferentes modelos existentes, se decidió utilizar la norma ISO 22301:2019 por ser una herramienta holística enfocada en una adecuada gestión de los riesgos. Este modelo está basado en el ciclo de Deming, que busca la calidad y mejora continua mediante sus cuatro etapas: planear, hacer, verificar y actuar. Además, la ISO 22301:2019 es una norma internacional que proporciona un marco de buenas prácticas para apoyar a las organizaciones en la gestión efectiva de sus operaciones ante el impacto de eventos disruptivos que las frenen. Esta norma está estructurada en diez cláusulas que sirven como referencia para el diseño de un SGCN, dado que contienen los objetivos, políticas, protocolos, procedimientos, matrices, mapas de procesos, listas y planes, entre otros documentos.

Es preciso mencionar que una organización, para implementar la ISO 22301, debe contratar el servicio de auditores e implementadores expertos en la herramienta, así como capacitar a todo el recurso humano que se verá involucrado en este sistema. Adicionalmente, hay que señalar que la certificación de la ISO es costosa y dura tres años.

\subsection{Gestión de riesgos}

Un riesgo es «la probabilidad de que la ocurrencia de un suceso adverso afecte a la organización e impacte en su habilidad para lograr sus objetivos estratégicos y por ende la capacidad de cumplir su misión y visión» (Cañas, 2009, p. 6). Para Narvaez (2009), el riesgo es

una condición latente que, al no ser modificada o mitigada a través de la intervención humana o por medio de un cambio en las condiciones del entorno físico-ambiental, anuncia un determinado nivel de impacto social y económico hacia el futuro, cuando un evento físico detona o actualiza el riesgo existente (p. 9).

La gestión de riesgos implica un conjunto de actividades encargadas de la dirección y el control de la organización frente al riesgo que tiene como objetivo la creación y proyección del valor (ISO 31000:2018). Para Cañas (2009), "la gestión de riesgos debe considerarse una función primordial y uno de los componentes claves a ser tomado en cuenta desde el proceso de planificación estratégica por todos los estamentos de la organización» (p. 3).

Un marco de evaluación de riesgos adecuado ayuda a las organizaciones a hacer planes de contingencia para dejar de perder recursos después de una ocurrencia de riesgo (Torabi et al., 2016), siendo uno de ellos un sistema de gestión de continuidad del negocio. Para el correcto funcionamiento de un sistema de gestión de la continuidad del negocio, se deben gestionar adecuadamente los riesgos, lo que implica identificarlos, analizarlos y valorarlos para, finalmente, idear una adecuada estrategia para el tratamiento de cada riesgo de acuerdo con su impacto. 
Existen distintas metodologías para gestionar los riesgos, una de ellas es el estándar australiano para la administración de riesgos AS/NZS 4360:1999. Esta metodología se enfoca, primero, en establecer el contexto de la organización y luego identificar, analizar, evaluar y tratar los riesgos. Se monitorea y se revisa el sistema de gestión de riesgos, a la par que se comunica y consulta a todos los stakeholders pertinentes durante todo el proceso. Esta metodología sirvió de base para las desarrolladas posteriormente (Cañas, 2009, pp. 13-15). Otra metodología es la ISO 31000, norma que cuenta con principios que permiten gestionar los riesgos eficientemente.

Para el diseño del SGCN se utilizó la ISO 31000:2018 como marco de referencia para la gestión de riesgos, debido a que brinda directrices para gestionar y analizar los riesgos correctamente. La ISO 31000 está estructurada en torno a tres elementos claves: nociones de la gestión de riesgos, marco de labores para la administración de riesgos y proceso de administración de riesgos (Lizarzaburu et al., 2017).

\subsection{Gestión por procesos}

La gestión por procesos es una eficaz herramienta de gestión que permite obtener mejores resultados en el menor tiempo posible y, por lo tanto, alcanzar la competitividad y la excelencia en el desempeño organizacional (Valdés Gutiérrez, 2015). Asimismo, permite comprender la configuración de los procesos del negocio, conocer sus fortalezas y debilidades, replantear los procesos que necesitan ser mejorados o rediseñados, establecer prioridades, iniciar planes de mejora con el objetivo de alcanzar las metas establecidas y eliminar las ineficiencias. Esto supone gestionar a los clientes y sus expectativas, las entradas y salidas de los procesos, y las actividades que generan valor a la organización (Medina, 2009).

Otro elemento importante en la gestión por procesos es el ciclo de Deming, más conocido como el ciclo de mejora continua PDCA (Plan, Do, Check, Act). Este ciclo es una herramienta trascendente para las organizaciones que se encuentran en busca de calidad y mejora continua. En la primera etapa, relativa al planeamiento, se determinan los objetivos a lograr, se realiza un diagnóstico de la situación actual de los procesos y se establecen los pasos a seguir a través de un plan de trabajo. De manera similar, en la etapa relacionada al hacer, se aplica el plan propuesto en la primera etapa; en la etapa relativa a la verificación, se comparan los resultados obtenidos contra los esperados; y, por último, en la etapa relacionada al actuar, se evalúan los resultados de la verificación previa, se corrige, y se estandarizan y sistematizan los procedimientos (Salas-Rueda, 2018, p. 10).

El enfoque en procesos resulta imprescindible para un sistema de continuidad del negocio, puesto que el establecimiento de un plan de continuidad de negocio parte de la identificación de los procesos clave para la creación de valor, así como de la interrelación que existe entre ellos (ISO, 2015).

\section{Metodología}

Respecto a la metodología, el alcance del presente documento es descriptivo; esto es, se enfoca en detallar características de procesos específicos sin establecer vínculos entre 
ellos con el fin de analizar el comportamiento de dichos procesos (Pasco \& Ponce, 2018, p. 43). A su vez, la delimitación del alcance se hace efectiva con la definición proporcionada, dado que el trabajo tiene como finalidad especificar las características del proceso del servicio de exportación que ofrece la agencia de carga con base en la ISO 22301:2019. Así también, el paradigma corresponde al de un trabajo analítico-descriptivo y de gestión aplicada, por lo que se han considerado tres aristas para el desarrollo del diseño que Pasco y Ponce recomiendan: el enfoque, la estrategia general y el horizonte temporal. Para empezar, el enfoque es cualitativo, puesto que durante el desarrollo del documento no se ha probado hipótesis alguna; por el contrario, se han generado y perfeccionado planes conforme los datos recabados se iban incrementando y, tal y como señalan Hernández et al. (2014), las hipótesis son un resultado del estudio realizado (p. 8). En ese sentido, la estrategia general escogida para la elaboración de la investigación es la de estudio de caso, dado que se ha investigado a profundidad un solo caso como un sistema que contiene partes integradas y límites establecidos; es decir, una agencia de carga ubicada en el Callao (Pasco \& Ponce, 2018, p. 60).

Para poder recabar la información necesaria, se dispusieron de dos técnicas de recolección: la entrevista y la lista de verificación. La primera hace referencia a las ocho entrevistas realizadas al personal de Ravmar a través de la plataforma virtual Meet, las cuales han sido distribuidas de la siguiente forma:

- Tres entrevistas estructuradas a la Alta Dirección.

- Dos entrevistas semiestructuradas al área operativa.

- Dos entrevistas semiestructuradas al área administrativa.

La segunda es la herramienta básica y necesaria para la implementación de todo sistema integrado de gestión, que además facilita y permite determinar el nivel de cumplimiento de Ravmar con respecto a los requisitos de la norma ISO 22301:2019.

No obstante, nada de lo anteriormente emitido sería posible sin haberse determinado una muestra correspondiente, por lo que esta investigación tiene dos tipos de muestra: muestra de expertos y muestra de casos tipo. Por un lado, la muestra de expertos ha sido necesaria para conseguir datos de calidad de expertos relacionados a la norma en mención, para lo cual se localizó a auditores e implementadores de la norma que, a su vez, manejaran los tres ejes temáticos detallados en el marco teórico. Por otro lado, la muestra de casos tipo realizada está alineada a las divisiones del grupo social presente en la agencia de carga; esto es, los trabajadores del área operativa y administrativa (Fernández \& Baptista, 2014, p. 387).

Para finalizar, el horizonte temporal es de tipo transversal, dado que el presente documento tiene como finalidad describir la realidad organizacional de Ravmar con información que la agencia de carga internacional ha proporcionado y que corresponde al periodo en el que fue realizada la presente investigación (Ponce \& Pasco, 2018, p. 49). 


\section{Discusión de resultados}

En el presente apartado se presenta la propuesta de diseño del SGCN para el servicio de exportación que ofrece Ravmar, acorde con los requisitos de la norma ISO 22301:2019. Para ello, se consideraron las siete cláusulas de la norma, que se agruparon con base al ciclo de Deming (Plan, Do, Check y Act) pues, como indican Figueroa y Salamanca (2013), la administración de todo sistema de gestión puede ser enmarcada bajo el ciclo en mención, que facilita la implantación programada, organizada y controlada del sistema, dado que el objetivo es la mejora continua (p. 17).

\subsection{Cuarta cláusula: contexto}

Nivel de cumplimiento previo: $7 \%$

El objetivo de esta cláusula es comprender a la organización y su contexto, así como las necesidades de los stakeholders pertinentes. Por consiguiente, se elaboró la matriz FODA, la matriz de partes interesadas y el documento del alcance del sistema de gestión, entre otros documentos.

\subsection{Quinta cláusula: liderazgo}

Nivel de cumplimiento previo: $0 \%$

La finalidad de esta cláusula es identificar la existencia del liderazgo y del compromiso por parte de la Gerencia de Ravmar hacia el sistema de gestión, así como designar los roles y las responsabilidades de los trabajadores que forman parte del alcance. Por esa razón, se elaboró la política del SGCN y la comunicación de la misma, el manual de organización y funciones (MOF), y la carta de compromiso de la Gerencia.

\subsection{Sexta cláusula: planificación}

Nivel de cumplimiento previo: $3 \%$

El fin de esta cláusula es establecer las acciones para abordar riesgos y oportunidades, encarar los objetivos de la continuidad del negocio y cumplir con la planificación para lograrlos. Por ende, se elaboró la matriz de riesgos y oportunidades, y la matriz de objetivos y metodología de riesgos, entre otros documentos.

\subsection{Séptima cláusula: soporte}

Nivel de cumplimiento previo: $0 \%$

El objetivo de esta cláusula es conocer cuáles son los recursos críticos necesarios para efectuar el servicio de exportación, así como garantizar la competencia de las partes interesadas pertinentes y su interiorización de la continuidad de negocio. Además, permite comprobar si existe un plan de comunicación para que los stakeholders correspondientes conozcan acerca de la compañía, para lo que se generó una matriz de administración de recursos, así como los planes de competencia, comunicación y concientización. 


\subsection{Octava cláusula: operación}

Nivel de cumplimiento previo: $0 \%$

La finalidad de esta cláusula es analizar el impacto al negocio generado por las fuentes de riesgo identificadas y proponer planes de continuidad de negocio para contrarrestar dichos eventos. Por ello, fueron elaborados la matriz de análisis de impacto del negocio (BIA, por sus siglas en inglés) y los planes de continuidad, contingencia, emergencias, recuperación de desastres, y ejercicios y pruebas.

\subsection{Novena cláusula: evaluación de desempeño}

Nivel de cumplimiento previo: $0 \%$

El fin de esta cláusula es monitorear, medir y analizar dichos planes establecidos, así como generar un programa de auditoría interna y de revisión por parte de la Gerencia. Para ello, se elaboró la matriz de evaluación y monitoreo de los planes, así como un programa anual de auditorías en el que se establecen los objetivos de la primera, las competencias requeridas para los auditores y la plantilla de evaluación, entre otros documentos.

\subsection{Décima cláusula: mejora}

Nivel de cumplimiento previo: $0 \%$

El objetivo de esta cláusula es proveer seguimiento a las acciones correctivas y/o no conformidades que resulten de los planes establecidos, así como el establecimiento de directrices para una evaluación y mejora continua. Por consiguiente, se realizó la matriz de mejora continua y un procedimiento de mejora o, en su defecto, acciones correctivas.

A continuación, se presenta el resumen de los documentos, las matrices y los planes elaborados, respetando los requisitos de las siete cláusulas anteriormente mencionadas (ver tabla 1).

Tabla 1. Documentos, matrices y planes elaborados

\begin{tabular}{l|l|l|l|l|l} 
Etapa & Cláusula & Requisito & $\begin{array}{l}\% \text { de cum- } \\
\text { plimiento } \\
\text { inicial }\end{array}$ & $\begin{array}{l}\% \text { de cum- } \\
\text { plimiento } \\
\text { final }\end{array}$ & $\begin{array}{l}\text { Información } \\
\text { documentada }\end{array}$ \\
\hline Plan & 4. Contexto & $\begin{array}{l}\text { 4.1. Comprender la } \\
\text { organización y su } \\
\text { contexto }\end{array}$ & $25 \%$ & $100 \%$ & $\bullet$ Matriz FODA \\
\cline { 2 - 5 } & $\begin{array}{l}\text { 4.2. Comprender las } \\
\text { necesidades y expec- } \\
\text { tativas de las partes } \\
\text { interesadas }\end{array}$ & $15 \%$ & $100 \%$ & $\begin{array}{l}\text { - Matriz de partes intere- } \\
\text { sadas pertinentes } \\
\text { Matriz legal }\end{array}$ \\
\cline { 2 - 5 } & $\begin{array}{l}\text { 4.3. Determinar el } \\
\text { alcance del sistema de } \\
\text { gestión de continuidad } \\
\text { del negocio }\end{array}$ & $25 \%$ & $100 \%$ & $\begin{array}{l}\text { - Documento de alcance } \\
\text { del SGCN }\end{array}$ \\
\cline { 2 - 5 } & $\begin{array}{l}\text { 4.4. Sistema de gestión } \\
\text { de continuidad del } \\
\text { negocio }\end{array}$ & $0 \%$ & $100 \%$ & $\begin{array}{l}\text { Mapa general de } \\
\text { procesos }\end{array}$ \\
\hline
\end{tabular}




\begin{tabular}{|c|c|c|c|c|c|}
\hline & \multirow[t]{3}{*}{ 5. Liderazgo } & $\begin{array}{l}\text { 5.1. Liderazgo y com- } \\
\text { promiso }\end{array}$ & $0 \%$ & $100 \%$ & $\begin{array}{l}\text { - Carta de compromiso } \\
\text { de la Alta Dirección }\end{array}$ \\
\hline & & 5.2. Política & $0 \%$ & $100 \%$ & $\begin{array}{l}\text { - } \text { Política del SGCN } \\
\text { - Comunicación de la } \\
\text { política del SGCN }\end{array}$ \\
\hline & & $\begin{array}{l}\text { 5.3. Roles, responsabili- } \\
\text { dades y autoridad }\end{array}$ & $0 \%$ & $100 \%$ & $\begin{array}{l}\text { - Manual de organización } \\
\text { y funciones }\end{array}$ \\
\hline & \multirow[t]{3}{*}{ 6. Planificación } & $\begin{array}{l}\text { 6.1. Acciones para } \\
\text { abordar riesgos y opor- } \\
\text { tunidades }\end{array}$ & $0 \%$ & $100 \%$ & $\begin{array}{l}\text { - Matriz de riesgos y } \\
\text { oportunidades } \\
\text { - Metodología de riesgos }\end{array}$ \\
\hline & & $\begin{array}{l}\text { 6.2. Objetivos de } \\
\text { continuidad del negocio } \\
\text { y planificación para } \\
\text { alcanzarlos }\end{array}$ & $15 \%$ & $100 \%$ & - Matriz de objetivos \\
\hline & & $\begin{array}{l}\text { 6.3. Planificación de } \\
\text { cambios en el sistema } \\
\text { de gestión de continui- } \\
\text { dad del negocio }\end{array}$ & $0 \%$ & $100 \%$ & $\begin{array}{l}\text { - Proceso de determina- } \\
\text { ción de cambios } \\
\text { - Detalle y seguimiento } \\
\text { de solicitudes de mejo- } \\
\text { ras y/o cambios }\end{array}$ \\
\hline & \multirow[t]{5}{*}{ 7. Soporte } & 7.1. Recursos & $0 \%$ & $100 \%$ & $\begin{array}{l}\text { - Administración de } \\
\text { recursos } \\
\text { - Matriz de análisis de } \\
\text { capacidad y demanda }\end{array}$ \\
\hline & & 7.2. Competencia & $0 \%$ & $100 \%$ & - Plan de competencia \\
\hline & & 7.3. Toma de conciencia & $0 \%$ & $100 \%$ & - Plan de concientización \\
\hline & & 7.4. Comunicación & $0 \%$ & $100 \%$ & - Plan de comunicación \\
\hline & & $\begin{array}{l}\text { 7.5. Información docu- } \\
\text { mentada }\end{array}$ & $0 \%$ & $100 \%$ & $\begin{array}{l}\text { - Gestión de la informa- } \\
\text { ción documentada } \\
\text { - Matriz de información } \\
\text { documentada del } \\
\text { SGCN } \\
\text { - Recursos críticos }\end{array}$ \\
\hline \multirow[t]{6}{*}{ Do } & \multirow[t]{6}{*}{ 8. Operación } & $\begin{array}{l}\text { 8.1. Planificación y } \\
\text { control operacional }\end{array}$ & $0 \%$ & $100 \%$ & $\begin{array}{l}\text { - Matriz de indicadores } \\
\text { del SGCN } \\
\text { - Mapa de procesos } \\
\text { - Flujograma de pro- } \\
\text { cesos }\end{array}$ \\
\hline & & $\begin{array}{l}\text { 8.2. Análisis de impacto } \\
\text { en el negocio y evalua- } \\
\text { ción de riesgos }\end{array}$ & $0 \%$ & $100 \%$ & $\begin{array}{l}\text { - Matriz de análisis de } \\
\text { impacto del negocio } \\
\text { (BIA) }\end{array}$ \\
\hline & & $\begin{array}{l}\text { 8.3. Estrategias y solu- } \\
\text { ciones de continuidad } \\
\text { del negocio }\end{array}$ & $0 \%$ & $100 \%$ & \multirow{2}{*}{$\begin{array}{l}\text { - Plan de continuidad del } \\
\text { negocio } \\
\text { - Plan de contingencia } \\
\text { - Plan de emergencias } \\
\text { - Plan de ejercicios y } \\
\text { - Pruebas } \\
\text { - Plan de recuperación } \\
\text { de desastres }\end{array}$} \\
\hline & & $\begin{array}{l}\text { 8.4. Planes y procedi- } \\
\text { mientos de continuidad } \\
\text { del negocio }\end{array}$ & $0 \%$ & $100 \%$ & \\
\hline & & $\begin{array}{l}\text { 8.5. Programa de } \\
\text { ejercicios }\end{array}$ & $0 \%$ & $100 \%$ & \multirow[t]{2}{*}{ - Programa de ejercicios } \\
\hline & & $\begin{array}{l}\text { 8.6. Evaluación de la } \\
\text { documentación y las } \\
\text { capacidades de la conti- } \\
\text { nuidad del negocio }\end{array}$ & $0 \%$ & $100 \%$ & \\
\hline
\end{tabular}




\begin{tabular}{|c|c|c|c|c|c|}
\hline \multirow[t]{3}{*}{ Check } & \multirow[t]{3}{*}{$\begin{array}{l}\text { 9. Evaluación } \\
\text { de desem- } \\
\text { peño }\end{array}$} & $\begin{array}{l}\text { 9.1. Monitoreo, } \\
\text { medición, análisis y } \\
\text { evaluación }\end{array}$ & $0 \%$ & $100 \%$ & $\begin{array}{l}\text { - Matriz de monitoreo, } \\
\text { medición, análisis y } \\
\text { evaluación del SGCN }\end{array}$ \\
\hline & & 9.2. Auditoría interna & $0 \%$ & $100 \%$ & $\begin{array}{l}\text { - Programa anual de } \\
\text { - Maditorías } \\
\text { riesgos del programa } \\
\text { anual de auditorías } \\
\text { - Plantilla de auditores } \\
\text { internos } \\
\text { - Competencias del } \\
\text { - } \text { auditor } \\
\text { - } \text { audriz de evaluación de } \\
\text { - interna de auditoría } \\
\text { - Informe de auditoría } \\
\text { - Acta de reunión de } \\
\text { apertura y cierre } \\
\text { de auditoría } \\
\text { - Acta de sesión }\end{array}$ \\
\hline & & $\begin{array}{l}\text { 9.3. Revisión por parte } \\
\text { de la Gerencia }\end{array}$ & $0 \%$ & $100 \%$ & $\begin{array}{l}\text { - Procedimiento de } \\
\text { revisión por parte de la } \\
\text { Gerencia }\end{array}$ \\
\hline \multirow[t]{2}{*}{ Act } & \multirow[t]{2}{*}{ 10. Mejora } & $\begin{array}{l}\text { 10.1. No conformidades } \\
\text { y acciones correctivas }\end{array}$ & $0 \%$ & $100 \%$ & $\begin{array}{l}\text { - Procedimiento de } \\
\text { acciones correctivas o } \\
\text { de mejora }\end{array}$ \\
\hline & & 10.2. Mejora continua & $0 \%$ & $100 \%$ & $\begin{array}{l}\text { - Matriz de mejora } \\
\text { continua }\end{array}$ \\
\hline
\end{tabular}

Fuente: elaboración propia.

Cada uno de los documentos que se encuentran detallados en la columna denominada "Información documentada» forma parte del manual de continuidad del negocio, que ya ha sido proporcionado a la Alta Dirección como parte del desarrollo de esta implementación.

\section{Análisis de viabilidad de la implementación}

\subsection{Análisis cuantitativo}

En la presente sección se procede a determinar la viabilidad económica de la implementación del sistema diseñado y, para ello, se necesita determinar, en primer lugar, los costos de la implementación. En respuesta a ello, la tabla 2 señala que el costo total es de S/ 14342.

Tabla 2. Costos por etapa del desarrollo de la implementación

\begin{tabular}{l|l|l} 
Etapa & Actividad & Costo \\
\hline Sensibilización & $\begin{array}{l}\text { Capacitación a la Gerencia y al personal operativo } \\
\text { y administrativo }\end{array}$ & S/ 1440 \\
\hline Diagnóstico & Diagnóstico, valoración y mejora & S/ 0 \\
\hline
\end{tabular}




\begin{tabular}{l|l|l}
\hline \multirow{2}{*}{ Planeación y organización } & Generación de infografías & S/32 \\
\cline { 2 - 3 } & $\begin{array}{l}\text { Capacitación a la Gerencia y al personal operativo } \\
\text { y administrativo }\end{array}$ & S/3450 \\
\cline { 2 - 3 } & Elaboración de información documentada & S/ 0 \\
\hline \multirow{2}{*}{ Implementación } & Adquisición de recursos detallados en planes & S/ 720 \\
\cline { 2 - 3 } & Ejecución de auditoría interna & S/ 100 \\
\cline { 2 - 3 } & Realización de ejercicios y pruebas & S/ 600 \\
\hline Certificación & Certificación de la casa acreditada & S/ 8000 \\
\hline Costo total & & S/ 14 342
\end{tabular}

Fuente: elaboración propia.

En segundo lugar, se debe hallar el valor actual neto (VAN) y la tasa interna de retorno (TIR) de la implementación, para lo cual se efectuó el flujo de caja después de la certificación, considerando un periodo de tres años. De esa manera, con una tasa de descuento de $25 \%$, según la información brindada por una ejecutiva de banca-empresa, se obtuvo un VAN de S/ 10 690,45 y una TIR de 71,77\%.

Finalmente, se procedió a analizar los resultados, con lo que se determinó que el índice de rentabilidad (IR) es de 1,75; es decir, la certificación de Ravmar en la norma ISO 22301:2019 es económicamente factible y viable.

\subsection{Análisis cualitativo}

En este acápite se determina la posibilidad de la implementación de la propuesta con la certificación correspondiente; por ende, es necesario cumplir con la siguiente secuencia: para comenzar, presentar el diseño del SGCN, por lo que se expone la finalidad de la implementación y los planes realizados en función a cada recurso crítico de las operaciones de Ravmar y sus respectivos escenarios de riesgo. Luego, detallar la viabilidad económica de la implementación con la presentación del flujo de caja incremental del escenario de implementación para un periodo de tres años debido a la vigencia de la certificación. Por último, esperar la decisión de la Gerencia. Cabe resaltar que la Gerencia decidió implementar el diseño propuesto; sin embargo, optó por obtener la certificación a partir del tercer año de la implementación. En respuesta a ello, se efectuó el flujo de caja incremental para un periodo de seis años, considerando la certificación en el tercer año; y se determinó que el VAN era de S/ 28 501,75, una cifra que admite la viabilidad de la implementación.

\section{Conclusiones}

Dado que la literatura revisada no presenta estudios similares al presente trabajo, es preciso enfatizar que la contribución teórica de este documento radica en proporcionar un modelo de aplicabilidad y otorgar visibilidad e información acerca de uno de los principales operadores logísticos ubicados en Lima: la agencia de carga Ravmar Freight Logistics S.A.C.

Cabe indicar que el diseño del SGCN para la agencia de carga Ravmar Freight Logistics S.A.C se realizó acorde a la norma ISO 22301:2019 con el objeto proveer 
continuidad a los procesos que conforman el servicio de exportación. Confrontado con este diseño, Ravmar obtuvo un $10 \%$ de nivel de cumplimiento de la implementación del SGCN respecto a los requerimientos y cláusulas revisadas. Este valor de cumplimiento pone de manifiesto que, antes de la propuesta del sistema de gestión, la agencia de carga no cumplía con los requisitos necesarios para una adecuada implementación de continuidad del negocio para el servicio de exportación.

Se consiguió el objetivo principal del documento, que es la realización del presente estudio. Asimismo, se elaboró y entregó el manual de continuidad del negocio a Ravmar, el cual incluye los mapas y las matrices, entre otros documentos que son requisito para el cumplimiento al $100 \%$ del SGCN; de esa manera, se aseguró la continuidad de los procesos que conforman el servicio de exportación ante la ocurrencia de eventos disruptivos.

Para que Ravmar obtenga la certificación de la norma ISO 22301:2019, entregada por alguna casa acreditadora, fue de suma importancia cumplir eficaz y eficientemente el plan propuesto en el manual de continuidad de negocio.

EI IR, por su parte, fue de 1,75 , lo que se traduce en que cada sol invertido por Ravmar se convertirá en S/ 1,75 de retorno. Esto señala que la implementación de este SGCN es económicamente viable al generar eficiencia y rentabilidad en el servicio de exportación de la agencia de carga Ravmar.

Si se aplica el diseño del SGCN, Ravmar, así como los gerentes y/o tomadores de decisiones de otras agencias de carga, podrán contar con suficiente información para mitigar los riesgos que interrumpen la continuidad de sus operaciones ante la ocurrencia de eventos disruptivos como los descritos en el presente artículo.

Es sumamente necesario que los policy makers generen políticas para que exista un plan de continuidad del negocio tanto para las agencias de carga como para los demás operadores logísticos, ya que de esta manera se asegura el flujo de bienes entre las partes interesadas pertinentes y, como consecuencia, no se afecta la continuidad de las organizaciones ni se incrementa la tasa de desempleo, entre otros beneficios.

El presente documento es, en efecto, la base de conocimiento que proporciona un marco de referencia para el análisis y la aplicabilidad del diseño respecto a la continuidad del negocio en operadores logísticos del mismo sector en futuras investigaciones. De cara a nuevas líneas de investigación, sería enriquecedor no solo realizar el diseño del SGCN, sino también implementar dicho sistema en la organización y aplicarlo a otros actores dentro del sector logístico. 


\section{bibliografía}

\section{Cañas, L.}

2009 Gestión de riesgos de negocio. Desarrollo e Implementación de Sistemas de Gestión de Riesgos. https://webquery.ujmd.edu. sv/siab/bvirtual/BIBLIOTECA\%20VIRTUAL/ REVISTAS/D/ADDOCBD0001557.pdf

\section{Castro, L.}

2013 Diseño de un Sistema de Gestión de Continuidad de Negocios (SGN) para la Reniec bajo la óptica de la norma ISO/ IEC 22301 [tesis de licenciatura, Pontificia Universidad Católica del Perú]. Lima. http://tesis.pucp.edu.pe/repositorio/ bitstream/handle/20.500.12404/5110/ CASTRO_LAURA_DISE\%c3\%910_ SISTEMA_GESTION_CONTINUIDAD_ NEGOCIOS_RENIEC_NORMA_ISO_ IEC_22301.pdf? sequence =1\&isAllowed=y

\section{Cordova, M., \& Taquia, 0.}

2018 Llegando últimos: la situación de los servicios logísticos en Perú, riesgos y oportunidades. Notas Académicas PUCP. https://repositorio.pucp.edu.pe/index/ handle/123456789/133286

\section{Cordova, M., \& Wong, L.}

2020 Competitividad en el Perú 20192020: una perspectiva de cadena de suministro. Notas Académicas PUCP. https://repositorio.pucp.edu.pe/index/ handle/123456789/170910

Figueroa, H., \& Salamanca, M.

2013 Guías para la implementación y auditoría de planes de continuidad de negocio desde la perspectiva de las normas ISO 22301, BS 25999, NTC 5722 y las prácticas profesionales del DRIl y de ISACA [tesis de licenciatura, Universidad Piloto de Colombia]. Bogotá, http://repository.unipiloto.edu.co/ bitstream/handle/20.500.12277/2621/

Trabajos\%20de\%20grado.

pdf? sequence $=1$ \&isAllowed $=y$

Frikha, G., Lamine, E., Kamissoko, D., Benaben, F., \& Pingaud. $\mathbf{H}$.

Toward a modeling Tool for Business

Continuity Management. IFAC-

PapersOnLine, 54(1), 1156-1161. https://doi. org/10.1016/j.ifacol.2021.08.136

\section{Hernández, R., Fernández, C., \&} Baptista, P.

2014 Metodología de la investigación (6ta ed.). México, D. F.: McGraw-Hill Interamericana. http://observatorio.epacartagena.gov.co/ wp-content/uploads/2017/08/metodologiade-la-investigacion-sexta-edicion. compressed.pdf

Lizarzaburu, G., Barriga Apuero, G., Noriega Febres, L. E., Lopez, L., \& Mejía, P. Y.

2017 Gestión de riesgos empresariales: Marco de Revisión ISO 31000. Universidad César Vallejo. https://hdl.handle. net/20.500.12692/37274

Medina, A., Nogueira, D., Hernández, A., \& Viteri, J.

2009 Relevancia de la Gestión por Procesos en la Planificación Estratégica y la Mejora Continua. Revista Eidos. https://www. researchgate.net/publication/319254319_ Relevancia_de_la_Gestion_por_Procesos_ en_la_Planificacion_Estrategica_y_la_ Mejora_Continua

Narvaez, L., Lavell, A., \& Pérez, G.

La Gestión del riesgo de desastres:un enfoque basado en procesos. Secretaría de la Comunidad Andina. 


\section{bibliografía}

https://repositorio.gestiondelriesgo.gov.co/

handle/20.500.11762/19759

\section{Olaya, J.}

2019

Estándar ISO 22301:2019 para Continuidad del Negocio.

\section{Pacheco-Mangas, J., De las} Olas, M., \& Hombrados-

Mendieta, I.

Resiliencia y cultura organizacional de los servicios sociales en la era de la digitalización. Prisma Social: revista de ciencias sociales, (29), 123-137. https://revistaprismasocial.es/article/ view/3603\#: :text=La\%20resiliencia\%20 organizacional\%2C\%20entendida \%20 como,de\%20su\%20funcionamiento\%20 y\%20cultura

\section{Pasco, M., \& Ponce, M.}

Guía de investigación en Gestión.

Lima: Vicerrectorado de Investigación

PUCP. https://cdn02.pucp.education/ investigacion/2016/06/16192132/Guia-deInvestigacion-en-Gestion_segunda-edicion.pdf

\section{Valdés Gutiérrez, E.}

Aplicación de Gestión por Procesos en una empresa importadora exportadora. Gestiopolis. https://www.gestiopolis.com/ aplicacion-de-gestion-por-procesos-en-unaempresa-importadora-exportadora/

\section{Quevedo, J.}

Revisión de modelos de Gestión de Continuidad del Negocio. Revista de Gestión de Sistemas e Informática, 9(1). https://pdfs.semanticscholar. org/8580/6386da6194e7053ddb1f7948f 6ca1f569098.pd

\section{Salas-Rueda, $\mathbf{R}$.}

2018

Uso del ciclo de Deming para asegurar la calidad en el proceso educativo sobre las Matemáticas. Ciencia Unemi, 11(27), 8-19. https://doi.org/10.29076/issn.25287737vol11iss27.2018pp8-19p

Torabi, S. A., Giahi, R., \& Sahebjamnia, N.

An enhanced risk assessment framework for business continuity management systems. Safety Science, 89, 201-218. https://doi.org/10.1016/j.ssci.2016.06.015

\section{UNE-ISO 31000}

Gestión del riesgo. Directrices. https:// www.iso.org/obp/ui\#iso:std:iso:31000:ed$2: \mathrm{v} 1: \mathrm{es}$

\section{World Bank Group} International LPI. https://lpi.worldbank.org/ international/global

\section{World Economic Forum (WEF)}

La difícil situación de Perú ilustra el peligro del COVID-19 para los países en desarrollo. https://www.weforum.org/agenda/2020/06/ the-plight-of-peru-illustrates-the-danger-ofcovid-19-to-developing-countries/

\section{World Health Organization (WHO)}

WHO Coronavirus (COVID-19) DashboardPeru. https://covid19.who.int/region/amro/ country/pe

Fecha de recepción: 26 de octubre de 2021 Fecha de aceptación: 2 de diciembre de 2021 Fecha de publicación: 4 de diciembre de 2021 